\section{Análise de custos da atenção hospitalar a recém- nascidos de risco: uma comparação entre Unidade Intermediária Convencional e Unidade Canguru}

\author{
Cost analysis of hospital care for newborns at risk: \\ comparison of an Intermediate Neonatal Care Unit \\ and a Kangaroo Unit
} Análisis de los costes de la atención hospitalaria
para los recién nacidos en riesgo: una comparación
entre la Unidad Intermedia Neonatal y la Unidad
Canguro
Aline Piovezan Entringer 1

Maria Auxiliadora de Sousa Mendes Gomes 1 Márcia Pinto 1

Rosângela Caetano 2

Cynthia Magluta 1

Zeni Carvalho Lamy 3

\author{
${ }^{1}$ Instituto Nacional de Saúde \\ da Mulher, da Criança e \\ do Adolescente Fernandes \\ Figueira, Fundação Oswaldo \\ Cruz, Rio de Janeiro, Brasil. \\ 2 Instituto de Medicina \\ Social, Universidade do \\ Estado do Rio de Janeiro, Rio \\ de Janeiro, Brasil. \\ ${ }_{3}^{3}$ Departamento de Saúde \\ Pública, Universidade \\ Federal do Maranhão, São \\ Luís, Brasil. \\ Correspondência \\ A. P. Entringer \\ Instituto Nacional de Saúde \\ da Mulher, da Criança e \\ do Adolescente Fernandes \\ Figueira, Fundação Oswaldo \\ Cruz. \\ Av. Rui Barbosa 716, Rio de \\ Janeiro, $R J$ \\ 22250-020, Brasil. \\ alineentringer@gmail.com
}

\begin{abstract}
The aim of this study was to compare the direct costs of implementation of the Kangaroo Method and an Intermediate Neonatal Care Unit, from the perspective of the Brazilian Unified National Health System (SUS) in Rio de Janeiro, Brazil. Newborns were eligible for inclusion if they were clinically stable and were able to receive care in those two modalities. A decision tree model was developed that incorporated baseline variables and costs into a hypothetical cohort of 1,000 newborns, according to the literature and expert opinions. Daily cost was BR\$343.53 for the second stage of the Kangaroo Unit and BR $\$ 394.22$ for the Intermediate Neonatal Care Unit. The total cost for the hypothetical cohort was $B R \$ 5,710,281.66$ for the second and third stages of the Kangaroo Unit and $R \$ 7,119,865.61$ for the Intermediate Neonatal Care Unit. The Intermediate Neonatal Care Unit cost 25\% more than the Kangaroo Unit. The study can contribute to decision-making in health, in addition to providing support for studies related to economic evaluation in neonatal health.
\end{abstract}

Kangaroo-Mother Care Method; Humanization of Assistance; Costs and Cost Analysis; Low Birth Weight Infant

\section{Resumo}

O objetivo deste estudo foi realizar uma análise comparativa entre o custo da Unidade Canguru e da Unidade Intermediária Convencional sob a perspectiva do SUS, no Município do Rio de Janeiro, Brasil. A população de referência é de recém-nascidos estáveis clinicamente, que podem receber assistência nas duas modalidades de cuidado. Um modelo de análise de decisão para uma coorte hipotética de mil recém-nascidos elegiveis foi elaborado para comparar os custos em cada estratégia avaliada. As probabilidades dos eventos e o consumo de recursos de saúde foram incorporados ao modelo com base na literatura e consulta a especialistas. O custo da diária foi de $R \$ 343,53$ para a $2 \underline{a}$ etapa da Unidade Cangurue de $R \$ 394,22$ para a Unidade Intermediária Convencional. O custo para a coorte hipotética foi de $R \$ 5.710 .281,66$ para a assistência na 2a e $3 \underline{a}$ etapa da Unidade Canguru e de R\$7.119.865,61 para a Unidade Intermediária Convencional. A Unidade Intermediária Convencional apresentou custos 25\% superiores aos da Unidade Canguru. O estudo pode contribuir para a tomada de decisão na área da saúde, além de fornecer subsídios para pesquisas relacionadas à avaliação econômica na área neonatal.

Método Mãe-Canguru; Humanização da Assistência; Custos e Análise de Custo; Recém-nascido de Baixo Peso 


\section{Introdução}

O Método Canguru foi criado na Colômbia, em 1978, com o objetivo de substituir recursos tecnológicos que eram escassos naquele país. A pouca disponibilidade de equipamentos resultava, muitas vezes, na ocupação de dois ou três recém-nascidos na mesma incubadora, contribuindo para infecção cruzada nas unidades neonatais. Naquele contexto, a aplicação da Unidade Canguru poderia diminuir as taxas de infecção hospitalar e causar impacto na morbimortalidade neonatal e infantil 1 .

No Brasil, o Método Canguru foi introduzido em um contexto de ampla discussão sobre a necessidade de humanização da assistência ao recém-nascido de baixo peso ao nascer e a importância dos cuidados maternos para a recuperação dos bebês. Com base nessas preocupações, o Método Canguru foi incorporado como política nacional em 1999, pela Norma de Atenção Humanizada ao Recém-nascido de Baixo Peso: Método Canguru 2 (AHRNBP-MC). Essa norma propõe a sua aplicação em três etapas. A primeira se inicia antes mesmo do nascimento da criança, com a identificação das gestantes de alto risco, orientações e suporte para a gestante e a família. A partir do nascimento do bebê e necessidade de internação na Unidade de Terapia Intensiva Neonatal (UTIN) ou Unidade Intermediária Convencional, a participação dos pais é estimulada, assim como o contato pele a pele com o recémnascido, de forma gradual e crescente, de maneira segura e agradável para ambos. A 2a etapa começa com a internação da díade mãe-bebê na Unidade Canguru. Para a participação nessa etapa, é necessário que o recém-nascido esteja estável clinicamente e que a mãe tenha desejo e disponibilidade para participar. A 3a etapa da Unidade Canguru se inicia após a alta hospitalar. Nessa etapa, o recém-nascido é assistido em visitas periódicas na maternidade de origem até alcançar $2.500 \mathrm{~g} 3$.

Ao longo desses anos, várias ações foram desenvolvidas visando à disseminação e ampliação de leitos de Unidade Canguru. Em 2012, pela Portaria no 930, de 10 de maio de 2012, que estabelece as diretrizes e objetivos para a organização da atenção integral e humanizada ao recém-nascido grave ou potencialmente grave, o Ministério da Saúde definiu novos padrões para os leitos neonatais, alterando a denominação referente ao cuidado intermediário, passando para a Unidade de Cuidados Intermediários Convencional e Unidade de Cuidados Intermediários Canguru 4.

A Unidade Canguru tem sido proposta como uma alternativa à Unidade Intermediária Convencional para bebês de baixo peso ao nascer es- táveis clinicamente. A segurança e a efetividade da Unidade Canguru têm sido apontadas por diversas evidências científicas, que sinalizam para diminuição de infecção relacionada à assistência à saúde, diminuição da apneia da prematuridade, estímulo ao aleitamento materno, controle térmico mais eficiente do recém-nascido, estímulo sensorial adequado, favorecimento do vínculo mãe e filho, melhora do desenvolvimento neurocomportamental do recém-nascido, melhora no relacionamento da família com a equipe de saúde, maior competência e confiança dos pais no manuseio do recém-nascido de baixo peso, inclusive após a alta hospitalar 5,6,7,8,9,10,11,12.

Um dos objetivos dos sistemas de saúde é incorporar tecnologias que forneçam o máximo de benefícios à população, com a garantia de que haverá recursos disponíveis para sua implementação. No entanto, ainda há uma insuficiência de estudos que estimem os custos de recémnascidos elegíveis nessa modalidade de cuidado. Alguns estudos apontam que o Método Canguru pode apresentar custos assistenciais inferiores aos da Unidade Intermediária Convencional $13,14,15$, mas são trabalhos realizados fora de nosso meio e, em alguns casos, possuem fragilidades metodológicas. Embora as análises de custos não se dediquem a examinar os resultados em saúde das intervenções, são etapas fundamental e intermediária para o desenvolvimento posterior de avaliações econômicas completas 16,17.

Por fim, há uma insuficiência de estudos desse tipo em nosso meio, particularmente no campo da assistência neonatal. Dessa forma, a análise dos custos de tais modalidades de cuidado neonatal pode se constituir em uma ferramenta importante, pois informa aos gestores o volume total dos recursos gastos com as intervenções e estima recursos necessários para sustentá-las e expandi-las 18, contribuindo para a tomada de decisão quanto à disseminação da 2a e 3 a etapa da Unidade Canguru enquanto uma alternativa à Unidade Intermediária Convencional para recém-nascidos estáveis clinicamente.

Considerando tais questões, o objetivo deste estudo é realizar uma análise de custos da Unidade Canguru e da Unidade Intermediária Convencional, na perspectiva do Sistema Único de Saúde (SUS) prestador da assistência neonatal na rede municipal de saúde do Rio de Janeiro.

\section{Método}

\section{Desenho do estudo}

Foi realizada uma análise comparativa de custos utilizando um modelo de decisão (árvore de de- 
cisão) que apresenta desfechos e custos relativos às 2a e 3 a etapas da Unidade Canguru e à Unidade Intermediária Convencional (Figura 1). Como mencionado, a la etapa da Unidade Canguru ocorre dentro da UTIN ou Unidade Intermediária Convencional e não constitui alternativa de cuidado à Unidade Intemerdiária Convencional, sendo, por essa razão, excluída da análise.

A análise de custos foi realizada na perspectiva do SUS prestador de serviços por intermédio da rede municipal de saúde do Rio de Janeiro.

Foram incluídas no estudo informações de custos de seis maternidades municipais do Rio de Janeiro que possuem administração direta da Secretaria Municipal de Saúde e Defesa Civil do Rio de Janeiro (SMSDC/RJ). As informações dessas maternidades foram incluídas porque elas apresentam Unidade Intermediária Convencional, Unidade Canguru e por atenderem a $47 \%$ da assistência perinatal do município do Rio de Janeiro, $60 \%$ dos nascimentos de recém-nascidos com muito baixo peso ao nascer e $67 \%$ dos nascimentos prematuros 19 .

O horizonte temporal examinado no modelo inicia-se com a estabilidade clínica do recémnascido e termina com a alta da 3a etapa da Unidade Canguru, alta da Unidade Intermediária Convencional ou óbito. Tendo em vista que este tempo não excede o período de um ano, não foi aplicada taxa de desconto às estimativas de custo 20.

O modelo simulou uma coorte hipotética de mil recém-nascidos elegíveis para a 2a etapa da Unidade Canguru ou Unidade Intermediária Convencional. A Unidade Intermediária Convencional permite a internação de recémnascidos com características distintas, sendo apenas uma parcela deles elegível à 2 a etapa da Unidade Canguru. Em contrapartida, todos os recém-nascidos elegíveis à 2a etapa da Unidade Canguru podem ser assistidos na Unidade Intermediária Convencional. A fim de homogeneizar a população em ambas as estratégias de cuidado, a população de referência do estudo foi definida como os recém-nascidos que atenderam aos critérios de elegibilidade para a 2a etapa da Unidade Canguru: estabilidade clínica, peso superior a $1.250 \mathrm{~g}$, nutrição enteral plena e permanência em ar ambiente sem relato de apneias que tenham necessitado de reanimação com oxigênio e pressão positiva nos últimos cinco dias 21 .

As intercorrências clínicas incluídas na árvore de decisão foram sepse, enterocolite necrotizante e apneia. A escolha dessas intercorrências se deu pela robustez da literatura encontrada em relação às mesmas 5,6,7,8,9,10,11,12. As probabilidades das intercorrências clínicas entre as modalidades de cuidado diferem, porém foi assumido no modelo que a gravidade da intercorrência é a mesma independentemente da unidade de origem do recém-nascidos e que as intercorrências são excludentes, isto é, o recém-nascido só apresenta uma intercorrência durante a internação.

Foram realizadas análises de sensibilidade univariada e bivariada para os parâmetros de maior incerteza. Para a variação dos parâmetros, foram utilizados os limites máximo e mínimo fornecidos durante a consulta aos especialistas ou pelos intervalos de confiança dos estudos que mediram a efetividade da Unidade Canguru em relação à probabilidade de ocorrência dos eventos de saúde.

Foram realizadas testagens dos valores internos incorporados ao modelo a fim de identificar erros e assegurar que os cálculos matemáticos foram realizados de forma acurada, garantindo a sua consistência.

\section{Obtenção das evidências}

\section{- Medidas de resultado}

Os dados utilizados na construção e alimentação da árvore de decisão se apoiaram na literatura e na consulta a especialistas da área neonatal 20,22. Foram consultados, de forma presencial e individual, seis especialistas, sendo três com formação na área médica e três em enfermagem, com mais de dez anos de experiência nas redes privada e/ ou pública de saúde do Rio de Janeiro. Os especialistas pertenciam a diferentes instituições de saúde e apenas alguns trabalhavam em hospitais que praticavam o Método Canguru.

As probabilidades de sepse na $2 a$ etapa da Unidade Canguru e na Unidade Intermediária Convencional e de apneia na 2 a etapa da Unidade Canguru foram obtidas do estudo de Silva et al. 8. Os demais parâmetros do modelo foram obtidos mediante consulta aos especialistas, pela impossibilidade de generalizar dados de estudos internacionais para a realidade brasileira.

\section{Obtenção dos custos}

A perspectiva do estudo determina o levantamento dos custos. Tratando-se da perspectiva do SUS provedor dos serviços, todos os recursos diretamente ligados à assistência hospitalar foram contabilizados 20,23. Foram incluídos os custos de internação na Unidade Intermediária Convencional, 2a etapa da Unidade Canguru e UTIN, já que os recém-nascidos assistidos nas duas primeiras unidades podem ter intercorrências clínicas, como apresentado no modelo de decisão, necessitando ocasionalmente de cuidado intensivo. 
Os custos de capital e os recursos humanos foram identificados e quantificados tomando por base a Política Nacional de Atenção ao Paciente Crítico (Portaria no 1.071 de 4 de julho de 2005) 24 para UTIN e Unidade Intermediária Convencional e na Norma de Orientação para a Implantação da Unidade Canguru (Portaria no 1.683, de 12 de julho de 2007) ${ }^{21}$ para a 2a etapa da Unidade Canguru.

O dimensionamento de recursos humanos foi realizado considerando o número de leitos disponíveis em cada maternidade e os seguintes pressupostos:

a) psicólogo: um profissional para cada maternidade, responsável por atender UTIN, Unidade Intermediária Convencional e 2a etapa da Unidade Canguru. Esse profissional contribui para as adaptações familiares, trabalhando para minimizar as diferenças entre o bebê imaginado pelos pais durante a gestação e o bebê real. Essa diferença é mais intensa quanto mais prematuro for o nascimento ou mais grave estiver o bebê. Dessa forma, o psicólogo dispensaria maior tempo com os recém-nascidos e os familiares da UTIN, seguido da Unidade Intermediária Convencional e 2a etapa da Unidade Canguru, tendo sido assumida uma distribuição da carga horária desse profissional de $50 \%$ das horas para UTIN, 30\% para Unidade Intermediária Convencional e $20 \%$ para a $2 \underline{a}$ etapa da Unidade Canguru;

b) responsável técnico, enfermeiro coordenador nutricionista e assistente social: foi considerado um profissional para cada maternidade, responsável por atender UTIN, Unidade Intermediária Convencional e 2a etapa da Unidade Canguru, assumindo-se igual proporção de horas para cada modalidade;

c) enfermeiro assistencial, fisioterapeuta, pediatra plantonista e pediatra diarista: foram utilizados os parâmetros estabelecidos pela Portaria MS no 1.071 de 4 de julho de 2005 para UTIN e Unidade Intermediária Convencional. A Portaria no 1.683 de 12 de julho de 2007 não descreve o quantitativo para a 2 a etapa da Unidade Canguru e, por essa razão, foi assumido esse leito como de Unidade Intermediária Convencional, considerando os mesmos parâmetros;

d) técnico de enfermagem: utilizados os parâmetros da Portaria MS no 1.071 de 4 de julho de 2005 para UTIN e Unidade Intermediária Convencional e Portaria no 1.683 de 12 de julho de 2007 para 2a etapa da Unidade Canguru;

e) fonoaudiólogo: não foi encontrada quantificação nas portarias, tendo sido utilizado o mesmo parâmetro que o fisioterapeuta. Foi considerado que o fonoaudiólogo atende aos recém-nascidos nos turnos da manhã e tarde, totalizando 12 ho- ras por dia de trabalho. Não foi considerado esse profissional no turno da noite.

Após a identificação e quantificação dos recursos humanos pelas portarias descritas, a média salarial das classes praticada pela SMSDC/ RJ foi utilizada para a valoração deles. Para a $3 \underline{a}$ etapa da Unidade Canguru, levou-se em conta o tempo gasto para cada consulta de 30 minutos, que foi calculado tendo por base o salário do pediatra.

A valoração dos custos de capital dos itens identificados e quantificados pelas portarias foi realizada com uso das bases oficiais do Sistema de Apoio à Elaboração de Projetos de Investimentos em Saúde (SomaSUS. http://portal. saude.gov.br/portal/saude/profissional/area. cfm?id_area $=1258$, acessado em 20/Out/2011) e Comprasnet (http://www.comprasnet.gov.br/, acessado em 06/Jan/2012). Para os equipamentos utilizados em mais de uma modalidade de cuidado, foi feito rateio simples. Os custos de construção do espaço físico foram considerados "custos afundados" (sunk costs) 25 e excluídos da análise.

Os custos de capital foram inseridos no modelo através da depreciação dos equipamentos. Para isso, foi considerada uma vida útil de vinte anos para os equipamentos e uma taxa de desconto de $5 \%$ 17,20. Foram adicionados a esse valor $10 \%$ do valor do equipamento, correspondentes à manutenção, como proposto pela SMSDC/RJ.

Os insumos necessários aos cuidados cotidianos e ao atendimento das intercorrências foram identificados e quantificados pela consulta aos especialistas, que estimaram os insumos utilizados pelos recém-nascidos em cada modalidade de cuidado e em caso de intercorrência clínica. Para a inclusão dos dados no estudo, foi utilizada a mediana da consulta aos especialistas. Foram selecionados os seguintes itens de custos: medicamentos e soluções, materiais hospitalares, dieta da mãe e do recém-nascido, gazes medicinais e exames laboratoriais. A valoração foi realizada com base nas informações da SMSDC/RJ e do Banco de Preços em Saúde (http://portal. saude.gov.br/portal/saude/Gestor/area.cfm?id_ area $=939$, acessado em 06/Jan/2012).

Os custos administrativos, como energia elétrica, água, vigilância, limpeza e conservação das unidades hospitalares, foram contabilizados mediante os gastos efetivamente realizados pelas maternidades e incluídos pelos contratos de cada uma delas. Foi realizado o rateio para cada centro de custo. O critério de rateio utilizado foi o metro quadrado $\left(\mathrm{m}^{2}\right)$ de área útil. No entanto, considerando que a UTIN e Unidade Intermediária Convencional são áreas críticas 26 , foram adicionados $40 \%$ do valor do $\mathrm{m}^{2}$ para os custos de 
energia elétrica e limpeza. Não foi possível utilizar o critério de rateio por $\mathrm{m}^{2}$ de área ponderada devido à indisponibilidade de dados dos demais centros de custos hospitalares nas maternidades. Para distribuição dos custos administrativos para cada recém-nascido, foi utilizado o número de leitos de cada centro de custo.

Os custos de capital foram variados nas análises de sensibilidade pelas informações do SomaSUS, que apresenta valor máximo e mínimo para a aquisição dos equipamentos. Para a variação de salários dos recursos humanos, somaram-se $30 \%$ a menos e a mais do caso-base para o valor mínimo e máximo, respectivamente.

Para a variação do valor dos insumos, utilizou-se o Banco de Preços em Saúde e a tabela unificada do SUS (Sistema de Gerenciamento da Tabela de Procedimentos, Medicamentos e OPM do SUS. http://sigtap.datasus.gov.br/tabelaunificada/app/sec/inicio.jsp, acessado em 06/ Jan/2012) e, quando não foram encontrados valores para variação nessas bases, usou-se como pressuposto uma variação de $50 \%$ a mais ou a menos do caso-base para valores máximos e mínimos, respectivamente.

Os custos referem-se ao ano de 2011 e foram expressos em Reais ( $\mathrm{R} \$$ ).

Para a elaboração do modelo de decisão e para os cálculos dos valores esperados, foi utilizado o software TreeAge Pro 2011 (Tree Age Software Inc., Williamstown, Estados Unidos).

$\mathrm{O}$ projeto de pesquisa que deu origem a este artigo foi aprovado pelo Comitê de Ética em Pesquisa (CEP) da SMSDC/RJ (protocolo 0013.0.314.008-11) e pelo CEP do Instituto Fernandes Figueira, Fundação Oswaldo Cruz (protocolo no 0028.0.008.314-11).

\section{Resultados}

\section{Diárias hospitalares}

Considerando o SUS prestador de serviços, todos os recursos utilizados para a prestação da assistência hospitalar dos recém-nascidos elegíveis foram identificados, quantificados e valorados. A Tabela 1 apresenta as diárias hospitalares e os itens de custos incluídos.

A distribuição dos itens de custo apurou que os recursos humanos foram responsáveis pela maior proporção da diária hospitalar, independentemente da modalidade de cuidado em que o recém-nascido se encontra.

\section{Análise do custo total}

Os custos do tratamento dos recém-nascidos elegíveis foram estimados para Unidade Intermediária Convencional e para a 2a e e 3a etapas da Unidade Canguru tendo em conta o custo das intercorrências clínicas incluídas no estudo (Figura 1). A árvore de decisão foi modelada com as probabilidades de intercorrência clínica em ambas as modalidades de cuidado e os custos foram estimados para uma coorte hipotética de mil recém-nascidos.

Os custos da $2 \underline{a}$ e da $3 \underline{a}$ etapas da Unidade Canguru juntas foram de $\mathrm{R} \$ 5.710 .281,66$ e os da Unidade Intermediária Convencional foram de $\mathrm{R} \$ 7.119 .865,61$.

Para o modelo de decisão desenvolvido, a Unidade Intermediária Convencional apresentou custo $25 \%$ superior às 2a e $3 \underline{a}$ etapas da Unidade Canguru para a coorte hipotética de mil recém-nascidos elegíveis durante a internação nas modalidades de cuidado avaliadas.

\section{Análise de sensibilidade}

Foi realizada análise de sensibilidade univariada separadamente para todos os parâmetros incluídos no modelo. Apenas quatro parâmetros influenciaram o modelo de decisão na análise univariada: dias de permanência na Unidade Intermediária Convencional, dias de permanência e valor da diária na 2a etapa da Unidade Canguru e o valor da diária na Unidade Intermediária Convencional. A variação para o máximo de dias na Unidade Canguru, mínimo de dias em Unidade Intermediária Convencional, valor máximo da diária na 2a etapa da Unidade Canguru e valor mínimo da diária na Unidade Intermediária Convencional contribuiu para que registrasse custos inferiores da Unidade Intermediária Convencional em comparação à 2a e 3a etapas da Unidade Canguru. Para os demais parâmetros o modelo se mostrou inalterado, com a Unidade Intermediária Convencional apresentando custos superiores às 2a $\mathbf{e} 3 \underline{a}$ etapas da Unidade Canguru.

Na análise de sensibilidade bivariada, isto é, com alteração simultânea de dois parâmetros do modelo sem modificação dos demais, a Unidade Intermediária Convencional apresenta custos inferiores aos das 2a e 3a etapas da Unidade Canguru. Quanto maior for o número de dias de internação na 2a etapa da Unidade Canguru e menor na Unidade Intermediária Convencional, maior o valor da diária na 2a etapa da Unidade Canguru e menor o valor da diária na Unidade Intermediária Convencional. 
Diária hospitalar dos recém-nascidos elegíveis para o estudo, segundo modalidade de cuidado, item de custo e variação. Rio de Janeiro, Brasil, 2011.

\begin{tabular}{|c|c|c|c|c|c|}
\hline & $\begin{array}{c}\text { 2a etapa da Unidade } \\
\text { Canguru [R\$ (mínimo } \\
\text { e máximo)] }\end{array}$ & $\begin{array}{c}\text { Unidade } \\
\text { Intermediária } \\
\text { Convencional }\end{array}$ & $\begin{array}{c}\text { UTIN } \\
\text { (apneia) }\end{array}$ & $\begin{array}{c}\text { UTIN } \\
\text { (enterocolite } \\
\text { necrotizante) } \\
\text { [R\$ (mínimo } \\
\text { e máximo)] }\end{array}$ & $\begin{array}{l}\text { UTIN (sepse) } \\
\text { [R\$ (mínimo } \\
\text { e máximo)] }\end{array}$ \\
\hline $\begin{array}{l}\text { Medicamentos e } \\
\text { soluções }\end{array}$ & $\begin{array}{c}1,03 \\
(0,73-2,02)\end{array}$ & $\begin{array}{c}1,23 \\
(0,73-2,02)\end{array}$ & $\begin{array}{c}4,67 \\
(2,96-8,86)\end{array}$ & $\begin{array}{c}144,16 \\
(76,86-224,59)\end{array}$ & $\begin{array}{c}67,32 \\
(34,50-102,87)\end{array}$ \\
\hline Materiais hospitalares & $\begin{array}{c}7,35 \\
(4,20-14,76)\end{array}$ & $\begin{array}{c}10,46 \\
(6,74-21,29)\end{array}$ & $\begin{array}{c}21,20 \\
(13,86-41,73)\end{array}$ & $\begin{array}{c}70,05 \\
(38,05-131,54)\end{array}$ & $\begin{array}{c}62,03 \\
(33,64-122,85)\end{array}$ \\
\hline $\begin{array}{l}\text { Dieta do recém- } \\
\text { nascido }\end{array}$ & $\begin{array}{c}9,46 \\
(4,73-14,19)\end{array}$ & $\begin{array}{c}15,86 \\
(7,93-23,79)\end{array}$ & $\begin{array}{c}7,91 \\
(3,96-11,87)\end{array}$ & $\begin{array}{c}5,41 \\
(2,71-8,12)\end{array}$ & $\begin{array}{c}6,24 \\
(3,12-9,37)\end{array}$ \\
\hline Dieta da mãe & $\begin{array}{c}31,80 \\
(15,90-47,70)\end{array}$ & $\begin{array}{c}31,80 \\
(15,90-47,70)\end{array}$ & - & - & - \\
\hline Exames laboratoriais & $\begin{array}{c}5,50 \\
(3,50-7,91)\end{array}$ & $\begin{array}{c}5,47 \\
(3,49-7,92)\end{array}$ & $\begin{array}{c}12,40 \\
(3,84-20,51)\end{array}$ & $\begin{array}{c}29,50 \\
(12,54-49,27)\end{array}$ & $\begin{array}{c}36,00 \\
(16,37-57,54)\end{array}$ \\
\hline Gazes medicinais & - & - & $\begin{array}{c}5,97 \\
(2,99-8,96)\end{array}$ & $\begin{array}{c}27,87 \\
(13,94-41,81)\end{array}$ & $\begin{array}{c}32,16 \\
(16,08-48,24)\end{array}$ \\
\hline $\begin{array}{l}\text { Depreciação dos } \\
\text { equipamentos }\end{array}$ & $\begin{array}{c}5,77 \\
(4,49-9,64)\end{array}$ & $\begin{array}{c}14,24 \\
(8,45-46,01)\end{array}$ & $\begin{array}{c}35,49 \\
(19,84-125,63)\end{array}$ & $\begin{array}{c}35,49 \\
(19,84-125,63)\end{array}$ & $\begin{array}{c}35,49 \\
(19,84-125,63)\end{array}$ \\
\hline $\begin{array}{l}\text { Custos } \\
\text { administrativos }\end{array}$ & $\begin{array}{c}15,86 \\
(7,93-23,79)\end{array}$ & $\begin{array}{c}10,16 \\
(5,08-15,24)\end{array}$ & $\begin{array}{c}16,67 \\
(8,33-25,00)\end{array}$ & $\begin{array}{c}16,67 \\
(8,33-25,00)\end{array}$ & $\begin{array}{c}16,67 \\
(8,33-25,00)\end{array}$ \\
\hline Recursos humanos & $\begin{array}{c}266,75 \\
(156,45-346,77)\end{array}$ & $\begin{array}{c}305,01 \\
(213,51-396,52)\end{array}$ & $\begin{array}{c}567,91 \\
(397,53-738,28)\end{array}$ & $\begin{array}{c}567,91 \\
(397,53-738,28)\end{array}$ & $\begin{array}{c}567,91 \\
(397,53-738,28)\end{array}$ \\
\hline $\begin{array}{l}\text { Total da diária } \\
\text { hospitalar }\end{array}$ & $\begin{array}{c}343,53 \\
(197,93-466,78)\end{array}$ & $\begin{array}{c}394,22 \\
(262,03-561,21)\end{array}$ & $\begin{array}{c}672,22 \\
(453,30-980,83)\end{array}$ & $\begin{array}{c}897,06 \\
(569,80-1.344,24)\end{array}$ & $\begin{array}{c}823,82 \\
(529,42-1.229,78)\end{array}$ \\
\hline
\end{tabular}

\section{Discussão}

A Unidade Canguru tem sido alternativa segura de cuidado neonatal para os recém-nascidos de baixo peso ao nascer estáveis clinicamente. Além de humanizar a assistência ao recém-nascido e sua família, a Unidade Canguru vem apresentando vantagens clínicas em relação ao cuidado convencional. Entretanto, várias dificuldades ainda são encontradas na sua disseminação da Unidade Canguru, entre elas a falta de recursos orçamentários para a expansão e avaliação da Unidade e a falta de recursos humanos 27,28.

No Brasil, como demonstrado nesta análise comparativa de custos, a Unidade Canguru apresentou custos inferiores ao da Unidade Intermediária Convencional para os recém-nascidos elegíveis. A análise realizada com o modelo de decisão analítico estimou custos $25 \%$ superiores para a Unidade Intermediária Convencional. Essa informação pode ser relevante para os processos de decisão relativos à expansão dessa modalidade neonatal de cuidados, ao lado dos benefícios apontados na literatura e em relatórios governamentais.

Na análise de sensibilidade univariada, o modelo se mostrou sensível a quatro parâmetros: valor da diária na 2a etapa da Unidade Canguru, valor da diária na Unidade Intermediária Convencional, dias de permanência na Unidade Intermediária Convencional e dias de permanência na 2a etapa da Unidade Canguru.

Os custos de ambas as modalidade de cuidado foram determinados pela quantificação e 


\section{Figura 1}

Representação esquemática da árvore de decisão.

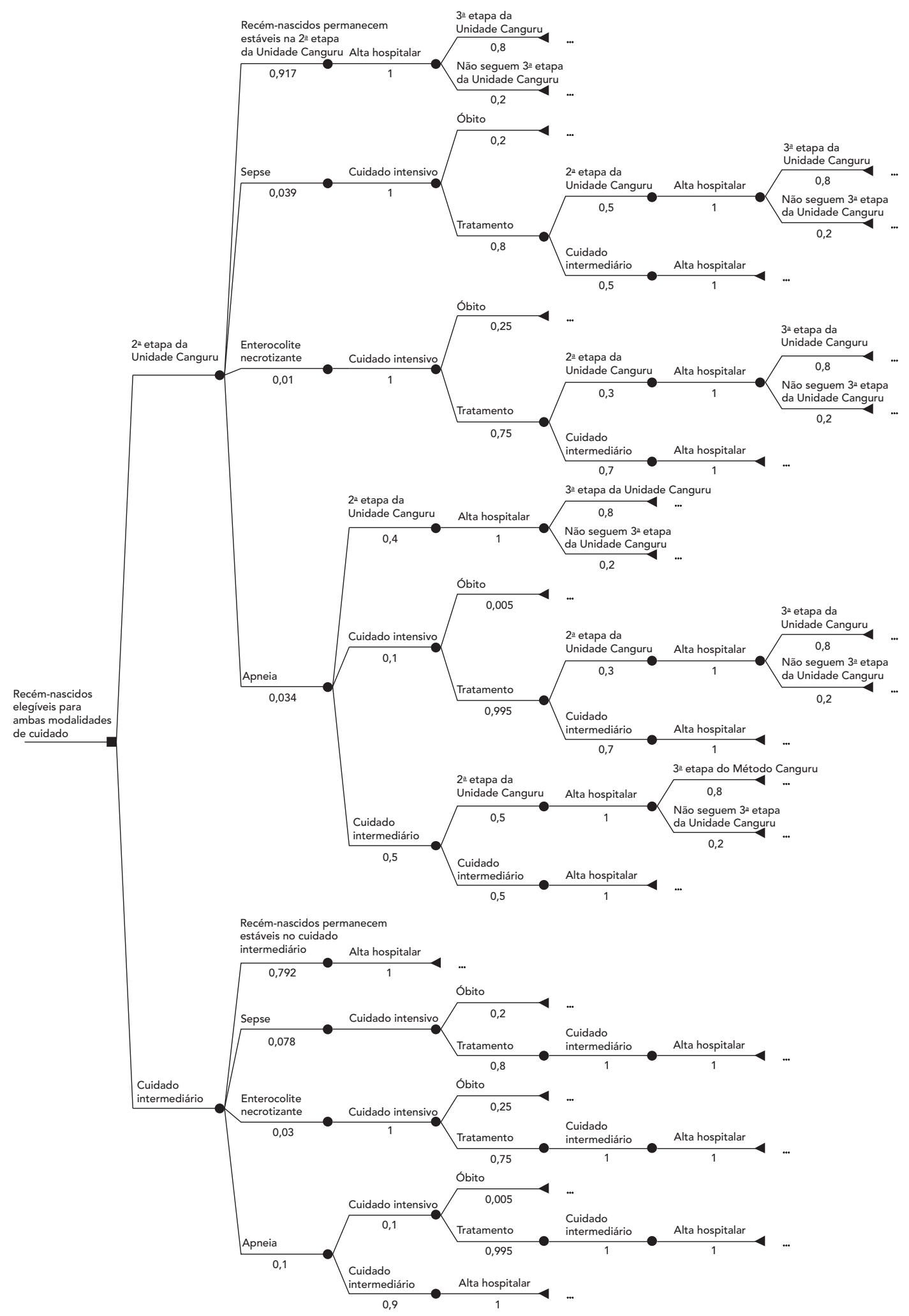


valoração dos recursos humanos, que representaram $77 \%$ dos custos na Unidade Intermediária Convencional e $78 \%$ na 2 a etapa da Unidade Canguru. Cabe ressaltar que o dimensionamento da quantidade de enfermeiros, pediatras plantonistas e diaristas, fonoaudiólogos e fisioterapeutas em ambas as unidades, considerou os mesmos parâmetros em razão da ausência de dimensionamento em normas e portarias. Mesmo utilizando esse pressuposto, o custo dos recursos humanos foi $14 \%$ superior para a Unidade Intermediária Convencional. Essa diferença entre as duas estratégias de cuidado foi determinada pelo quantitativo de técnicos de enfermagem.

$\mathrm{O}$ componente de custo relativo à dieta dos recém-nascidos diferiu entre as duas modalidades de cuidado em decorrência da maior prevalência de aleitamento materno na $2 \underline{a}$ etapa da Unidade Canguru. Assim, os recém-nascidos nessa modalidade de cuidado consomem menor quantidade de fórmula infantil, reduzindo a participação desse item de custo. $\mathrm{O}$ item de custo relativo à alimentação das mães foi incluído em ambas as modalidades de cuidado, pois a permanência delas também é permitida e estimulada na Unidade Intermediária Convencional.

O componente de custo referente à depreciação dos equipamentos foi maior na Unidade Intermediária Convencional. Isso se deve à maior densidade tecnológica nessa unidade, que se faz necessária para atender aos recém-nascidos de diversas características. $\mathrm{O}$ custo dos exames laboratoriais foi similar nas duas modalidades de cuidado. Já os custos com materiais hospitalares, medicamentos e soluções foram menores na $2 \underline{a}$ etapa da Unidade Canguru, pois como a mãe cuida do recém-nascido a maior parte do tempo e a prevalência de aleitamento materno é maior, o uso de luvas, seringas, copos, água destilada, entre outros materiais hospitalares, é menor na 2 etapa da Unidade Canguru quando comparado à Unidade Intermediária Convencional.

Os custos administrativos se mostraram superiores na 2a etapa da Unidade Canguru, já que as unidades destinadas a essa etapa são amplas, a fim de permitir a permanência da mãe junto ao recém-nascido durante o dia e a noite.

Destaca-se que, com a implantação da Unidade Canguru, não há a indicação de desativação dos leitos de Unidade Intermediária Convencional, uma vez que a Unidade Canguru só é indicada para um subgrupo de recém-nascidos elegíveis para esse tipo de cuidado, permanecendo a Unidade Intermediária Convencional como locus de cuidados para diversos outros bebês ainda não estáveis ou com outras condições patológicas ou cujas mães não tenham condições de permanecer nos hospitais junto a seus recém-nascidos. Contudo, a transferência dos recém-nascidos da Unidade Intermediária Convencional para a Unidade Canguru aumenta a rotatividade de leitos neonatais, questão importante em nosso país, que convive com carência e má distribuição regional de leitos para o cuidado a recém-nascidos 29

Por conta da escassez de estudos sobre a temática de custos aqui discutida, a comparação do presente estudo com outras referências é limitada, ainda mais que os poucos existentes, com uma única exceção, foram realizados em contextos externos, com estruturas assistenciais e de custos diferentes do nosso país. Portanto, discutimos a seguir os achados desta pesquisa com três estudos encontrados que descrevem custos em seus resultados.

Sloan et al. 13, em ensaio clínico randomizado realizado em Quito (Equador) com recém-nascidos menores que $2.000 \mathrm{~g}$ sem malformações, alterações respiratórias, metabólicas ou infecções, encontraram custo superior de US\$340 para a Unidade Intermediária Convencional quando comparado ao Método Canguru. Porém, o estudo não traz os custos relativos a cada modalidade de cuidado avaliada. Em outro ensaio clínico randomizado, realizado em três países, incluindo recém-nascidos entre $1.000 \mathrm{~g}$ e $1.999 \mathrm{~g}$, sem uso de oxigênio, fluidos endovenosos e excluindo os recém-nascidos malformados, Cattaneo et al. 14 evidenciaram que a Unidade Intermediária Convencional apresentou custos $50 \%$ maiores que o Método Canguru durante um ano de estudo. Foram incluídos no estudo 149 recém-nascidos para o Método Canguru e 136 para a Unidade Intermediária Convencional. Foram avaliados custos com recursos humanos (US $\$ 11.788$ e US $\$ 29.888$ para o Método Canguru e Unidade Intermediária Convencional, respectivamente) e outros custos como alimentação para mães e bebês, medicamentos e outros suplementos, raio-x, exames laboratoriais, lavanderia, roupas, combustível, eletricidade e manutenção dos equipamentos (US\$ 7.501 e US\$ 9.876 para Método Canguru e Unidade Intermediária Convencional, respectivamente).

Deve ser considerado que o estudo de Sloan et al. 13 foi desenvolvido no Equador e o trabalho de Cattaneo et al. 14 foi um estudo multicêntrico realizado no México, Indonésia e Etiópia, países em desenvolvimento onde o Método Canguru era utilizado em um contexto diferente do preconizado hoje no Brasil.

Lima et al. 15, por meio de um estudo descritivo em Recife (Pernambuco, Brasil), avaliaram recém-nascidos com peso entre $1.000 \mathrm{~g}$ e $1.750 \mathrm{~g}$, estáveis clinicamente, e encontraram custos três vezes maiores para a Unidade Intermediária 
Convencional. Foram incluídos custos de recursos humanos, diária hospitalar, materiais hospitalares, medicação e gazes, identificando um custo médio mensal de US $\$ 600$ para um bebê no Método Canguru e de US\$2.000 para um bebê na Unidade Intermediária Convencional.

O presente estudo encontra resultados similares no que diz respeito ao maior custo para a Unidade Intermediária Convencional e também a grande proporção de gastos com recursos humanos. Todavia, a diferença de custos entre as modalidades de cuidado, encontrada pelos autores citados, foi superior ao encontrado no presente estudo.

Cumpre, ainda, destacar que a presente investigação utiliza o método de modelagem para a aferição dos custos, delineamento diferente dos estudos utilizados para a comparação, os quais aferiram custos da prática local. Por fim, os trabalhos mencionados não apresentaram o caminho metodológico utilizado para o levantamento dos custos, limitando, dessa forma, as comparações.

As análises de custos feitas no Brasil devem ser adequadas à realidade nacional, de modo a não simplesmente importar dados que não sejam condizentes com o contexto local, uma das limitações da análise realizada decorre da escassez de estudos nacionais que discorram sobre a efetividade das intervenções e da utilização da Unidade Canguru no contexto em que ele é aplicado no país. O Método Canguru, em muitos países, é utilizado em recém-nascidos instáveis clinicamente e não apresenta as etapas padronizadas como no Brasil. Tal diferença de cenário exigiu a necessidade de consulta a especialistas para a definição de alguns dados de efetividade utilizados.

A consulta aos especialistas é um método de busca de informações que pode ser empregado quando lacunas são encontradas em relação à falta de dados na literatura ou quando se deseja, de alguma maneira, contemplar possíveis diferenças entre os dados da literatura e a realidade local 20,22. Vale lembrar, entretanto, que esse método possui algumas desvantagens, visto que informações advindas de especialistas podem representar convicções e experiências concretas da prática clínica. Buscou-se minimizá-las ampliando a consulta a seis especialistas, de ampla experiência e com formações acadêmicas diversas, atuantes em unidades assistenciais com e sem o Método Canguru, e utilizanda Unidade de coleta de informações presenciais, que permitia explicar e dirimir eventuais dúvidas em relação à aplicação dos questionários.

Também foi necessária a agregação de dados de custo de diferentes fontes de informação, devido à dificuldade em encontrar os custos dos itens incluídos no estudo em bases públicas de dados.

Ainda, outras limitações na realização do estudo devem ser mencionadas. A depreciação dos equipamentos considerou o número de leitos em cada modalidade de cuidado. Não foi possível realizar a depreciação dos equipamentos pela quantidade de recém-nascidos que utilizaram as unidades, pois os registros de internações mensais e/ou anuais na 2a etapa da Unidade Canguru estavam indisponíveis na SMSDC/RJ. O município em questão disponibilizou registros apenas para Unidade Intermediária Convencional e UTIN.

Para os custos administrativos, não foi possível realizar rateio por metro quadrado ponderado por causa da indisponibilidade de registros sobre os centros de custos hospitalares nas maternidades estudadas. Por essa razão, com as informações disponibilizadas pela SMSDC/RJ, foi possível realizar o rateio por metro quadrado de área útil. Buscando minimizar o problema, usouse como pressuposto que a limpeza e a utilização de energia elétrica são mais intensas em UTIN e Unidade Intermediária Convencional, por serem consideradas áreas críticas.

Outro aspecto a ser destacado diz respeito ao dimensionamento de recursos humanos. As normas e portarias do Ministério da Saúde não informam com precisão o quantitativo de alguns profissionais necessários às modalidades de cuidado, em especial para a 2a etapa da Unidade Canguru, o que dificultou a aferição dos custos.

As informações sobre custos hospitalares no setor público de saúde no Brasil ainda são incipientes e obstáculos são encontrados no momento de sua aferição. No entanto, com o intuito de minimizar essas questões, o trabalho foi realizado com uma modelagem que se baseou em portarias, manuais e especialistas, a fim de obter um padrão de assistência compatível com as práticas clínicas recomendadas. Essa opção possibilita uma estimativa de custos mais próxima ao padrão proposto, já que minimiza as variações de custos decorrentes de diferenças ou mesmo inadequações na prática assistencial concreta. Acredita-se que as informações sobre o custo estimado de uma prática assistencial definida por melhores evidências e diretrizes clínicas sejam essenciais à organização e programação dos serviços na perspectiva dos gestores, mas reforça-se a importância de desenvolvimento de estudos posteriores que examinem os diferenciais de custo levando em conta as práticas como efetivamente vêm sendo implementadas.

A perspectiva do estudo considerou o SUS provedor de serviços por intermédio da SMSDC/ RJ. Dessa forma, foram incluídos os custos dire- 
tamente relacionados à assistência à saúde. Não foram considerados os custos incorridos pelas famílias, como o tempo dos familiares, transporte, entre outros. Outras análises econômicas, considerando a perspectiva da sociedade e que inclua tais custos são importantes.

Além disso, a perspectiva de um provedor específico de serviços determina que os parâmetros utilizados reflitam a estrutura desse sistema local. Os parâmetros de custo utilizados no estudo foram baseados nas informações fornecidas pela SMSDC/RJ, sempre que disponível, já os parâmetros de efetividade foram baseados na opinião de especialistas dessa região. Logo, existem limitações para realizar generalizações para locais com parâmetros que se distinguem dos utilizados neste estudo. Novamente, reitera-se a necessidade de mais trabalhos sobre a temática em outras localidades brasileiras, bem como a importância de desenvolvimento de sistemas de custeio em nossas unidades, que possam informar de forma mais precisa os custos efetivamente incorridos, permitindo gerar dados que possam ajudar nas decisões sobre essas modalidades de cuidado em nosso país.

\section{Considerações finais}

As avaliações econômicas em saúde agregam valor e qualificam a tomada de decisão nos sistemas de saúde. Em vários países, essas avaliações são utilizadas para a incorporação e avaliação de tecnologias no sistema de saúde. No Brasil, no entanto, ainda são incipientes na área neonatal.
Assim, as informações contidas neste estudo, além de subsidiar os gestores na organização do cuidado ao recém-nascido no Brasil, podem contribuir para pesquisadores de diferentes áreas, particularmente nos campos da atenção neonatal e da avaliação econômica.

A melhoria da atenção ao recém-nascido e a redução da mortalidade neonatal estão definidos como eixos prioritários das ações do governo brasileiro. Dessa forma, a ampliação de leitos para o cuidado neonatal tem sido adotada em diferentes regiões do país, em paralelo a esforços com a oferta de cuidados mais humanizados e que sejam capazes de induzir autonomia de mães e familiares. Este estudo identificou que a Unidade Canguru possui custos inferiores à Unidade Intermediária Convencional para o grupo de recém-nascidos elegíveis. Seus resultados podem contribuir para o processo decisório em relação às diferentes modalidades do cuidado neonatal e podem ser de grande relevância para o alcance dos objetivos propostos de uma melhor e mais equânime atenção à população infantil.

\section{Resumen}

El objetivo de este estudio fue realizar un análisis comparativo entre el coste de la Unidad Canguro y la Unidad Intermedia Convencional, dentro de la perspectiva del Sistema Único de Salud (SUS) en el municipio de Río de Janeiro, Brasil. La población de referencia son los recién nacidos clínicamente estables, que pueden recibir asistencia en ambas modalidades de atención. Se diseñó un modelo de análisis de decisión para una cohorte hipotética de 1.000 bebés elegibles, con el fin de comparar los costes de cada estrategia evaluada. Las probabilidades de ocurrencias y consumo de recursos sanitarios se incorporaron al modelo de la literatura la consulta con expertos. El coste diario fue de $R \$ 343,53$ en la 2 a etapa de la Unidad Canguro y R\$394,22 en la Unidad Intermedia Convencional. El coste de la cohorte hipotética fue $R \$ 5,710,281.66$ para la asistencia en la segunda y tercera etapa de la Unidad Canguro y $R \$ 7,119,865.61$ para la Unidad Intermedia Convencional. La Unidad Intermedia Convencional tiene costes un 25\% más altos que la Unidad Canguro. El presente estudio puede contribuir a la toma de decisiones en el cuidado de la salud.

Metodo Madre-Canguro; Humanización de la Atención; Costos y Análissi de Costo; Recién Nacido de Bajo Peso 


\section{Colaboradores}

A. P. Entringer, M. A. S. M. Gomes e M. Pinto participaram da concepção do projeto e da redação das versões inicial e final do artigo. R. Caetano, C. Magluta e Z. C. Lamy participaram da revisão do projeto e da aprovação final da versão a ser publicada.

\section{Referências}

1. World Health Organization. Kangaroo mother care: a pratical guide. Geneva: World Health Organization; 2003

2. Ministério da Saúde. Norma de atenção humanizada do recém-nascido de baixo-peso: Método Mãe-Canguru. Brasília: Ministério da Saúde; 1999.

3. Área de Saúde da Criança, Secretaria de Atenção à Saúde, Ministério da Saúde. Atenção humanizada ao recém-nascido de baixo peso: Método Canguru. Brasília: Ministério da Saúde; 2009.

4. Ministério da Saúde. Portaria no 930, de 10 de maio de 2012. http://bvsms.saude.gov.br/bvs/saudele gis/gm/2012/prt0930_10_05_2012.html (acessado em 18/Dez/2012).

5. Conde-Agudelo A, Diaz-Rossello JL, Belizan JM. Kangaroo mother care to reduce morbidity and mortality in low birthweight infants. Cochrane Database Syst Rev 2011; (3):CD002771.

6. Lawn JE, Mwansa-Kambafwile J, Horta BL, Barros FC, Cousens S. "Kangaroo mother care" to prevent neonatal deaths due to preterm birth complications. Int J Epidemiol 2010; 39:i144-54.

7. Worku B, Kassie A. Kangaroo mother care: a randomized controlled trial on effectiveness of early kangaroo mother care for the low birthweight infants in Addis Ababa, Ethiopia. J Trop Pediatr 2005; 51:93-7.

8. Silva AAM, Lamy Filho F, Gomes MASM, Moreira MEL, Lamy ZC. Avaliação dos resultados do cuidado neonatal sob a perspectiva da Atenção Humanizada ao Recém-nascido de Baixo Peso - Método Canguru. http://portal.saude.gov.br/portal/arqui vos/pdf/relatorio_metodocanguru.pdf (acessado em 24/Mar/2011)
9. Renfrew MJ, Dyson L, McCormick F, Misso K, Stenhouse E, King SE, et al. Breastfeeding promotion for infants in neonatal units: a systematic review. Child Care Health Dev 2009; 36:165-78.

10. Moore ER, Anderson GC, Bergman N. Early skinto-skin contact for mothers and their healthy newborn infants. Cochrane Database of Syst Rev 2007; (3):CD003519.

11. Feldman R, Eidelman AI, Sirota L, Weller A. Comparison of skin-to-skin (Kangaroo) and traditiona care: parenting outcomes and preterm infant development. Pediatrics 2002; 110:16-26.

12. Ohgi S, Fukuda M, Moriuchi H, Kusumoto T, Akiyama T, Nugent JK, et al. Comparison of Kangaroo Care and Standard Care: behavioral organization, development, and temperament in healthy, lowbirth-weight infants through 1 year. J Perinatol 2002; 22:374-9.

13. Sloan NL, Leon Camacho LW, Rojas EP, Stern C; Maternidad Isidro Ayora Study team. Kangaroo mother method: randomised controlled trial of an alternative method of care for stabilized low-birth weight infants. Lancet 1994; 344:782-5.

14. Cattaneo A, Davanzo R, Worku B, Surjono A, Echeverria M, Bedri A, et al. Kangaroo mother care for low birthweight infants: a randomised controlled trial in different settings. Acta Paediatr 1998; 87:976-85.

15. Lima G, Quintero-Romero S, Cattaneo A. Feasibility, acceptability and cost of kangaroo mother care in Recife, Brazil. Ann Trop Paediatr 2000; 20:22-6.

16. Walker D. Cost and cost-effectiveness guidelines: which ones to use? Health Policy Plan 2001; 16: 113-21. 
17. Drummond MF, Sculpher MJ, Torrance GW O’Brien BJ, Stoddart GL. Methods for the economic evaluation of health care programmes. $3^{\text {rd }} \mathrm{Ed}$. New York: Oxford University Press; 2005.

18. Departamento de Economia da Saúde, Secretaria de Ciência, Tecnologia e Insumos Estratégicos, Ministério da Saúde. Programa Nacional de Gestão de Custos: manual técnico de custos. Conceitos e metodologia. Brasília: Ministério da Saúde; 2006. (Série A. Normas e Manuais Técnicos).

19. Gomes MASM, Lopes JMA, Moreira MEL, Gianini NOM. Assistência e mortalidade neonatal no setor público do Município do Rio de Janeiro, Brasil: uma análise do período 1994/2000. Cad Saúde Pública 2005; 21:1269-77.

20. Departamento de Ciência e Tecnologia, Secretaria de Ciência, Tecnologia e Insumos Estratégicos, Ministério da Saúde. Diretrizes metodológicas: estudos de avaliação econômica de tecnologias em saúde. Brasília: Ministério da Saúde; 2009. (Série A. Normas e Manuais Técnicos).

21. Ministério da Saúde. Portaria no 1.683, de 12 de julho de 2007. http://bvsms.saude.gov.br/bvs/sau delegis/gm/2007/prt1683_12_07_2007.html (acessado em 25/Out/2010).

22. Weinstein MC, O’Brien B, Hornberger J, Jackson J, Johannesson M, McCabe C, et al. Principles of good Practice for Decision Analytic Modeling in Health-Care Evaluation: Report of the ISPOR Task Force on Good Reasearch Practices - Modeling Studies. Value Health 2003; 6:9-17.

23. Vianna CMM, Caetano R. Avaliações econômicas como um instrumento no processo de incorporação tecnológica em saúde. Cad Saúde Colet (Rio J.) 2005; 13:747-66.
24. Ministério da Saúde. Portaria MS 1.071, de 04 de julho de 2005. Política Nacional de Atenção ao Paciente Crítico. http://www.sobrati.com.br/ms politica-critico.htm (acessado em 23/Out/2011)

25. Iunes RF. A concepção econômica de custos. In: Piola SF, Vianna SM, organizadores. Economia da saúde: conceitos e contribuições para a gestão da saúde. Brasília: Instituto de Pesquisa Econômica Aplicada; 1995. p. 227-48.

26. Asakura MK. Classificação de áreas em serviços de saúde. In: Agência Nacional de Vigilância Sanitária, organizador. Segurança do paciente em servi ços de saúde: limpeza e desinfecção de superfícies. Brasília: Agência Nacional de Vigilância Sanitária; 2010. p. 19-22.

27. Gontijo TL, Meireles AL, Malta DC, Proietii FA, Xavier CC. Avaliação da implantação do cuidado humanizado aos recém-nascidos com baixo peso - Método Canguru. J Pediatr (Rio J.) 2010; 86:33-9.

28. Colameo AJ, Rea MF. O Método Mãe Canguru em hospitais públicos do Estado de São Paulo, Brasil: uma análise do processo de implantação. Cad Saúde Pública 2006; 22:597-607.

29. Barbosa AP, Cunha AJLA. Neonatal and pediatric intensive care in Rio de Janeiro State, Brazil: an analysis of bed distribution, 1997 and 2007. Cad Saúde Pública 2011; 27 Suppl 2:S263-71.

Recebido em 17/Jul/2012

Versão final reapresentada em 23/Jan/2013 Aprovado em 01/Fev/2013 